\title{
Efficient retrovirus-mediated transfer of cell-cycle control genes to transformed cells
}

B.E. Strauss and

E. Costanzi-Strauss
Departamento de Histologia e Embriologia, Instituto de Ciências Biomédicas,

Universidade de São Paulo, São Paulo, SP, Brasil

\section{Correspondence}

B.E. Strauss

Departamento de Histologia e

Embriologia, ICB, USP

Av. Prof. Lineu Prestes, 1524

05508-900 São Paulo, SP

Brasil

Fax: + 55-11-818-7402

E-mail: bstrauss@usp.br

Presented at the I International Symposium on "Signal Transduction

and Gene Expression in Cell

Proliferation and Differentiation",

São Paulo, SP, Brasil,

August 31-September 2, 1998.

Publication supported by FAPESP.

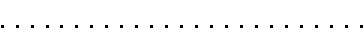

Received November 26, 1998 Accepted January 15, 1999

\section{Abstract}

The use of gene therapy continues to be a promising, yet elusive, alternative for the treatment of cancer. The origins of cancer must be well understood so that the therapeutic gene can be chosen with the highest chance of successful tumor regression. The gene delivery system must be tailored for optimum transfer of the therapeutic gene to the target tissue. In order to accomplish this, we study models of G1 cell-cycle control in both normal and transformed cells in order to

Key words - Gene therapy

- Clinical trial

- Retrovirus

- Animal model

- Cell cycle

- Growth arrest understand the reasons for uncontrolled cellular proliferation. We then use this information to choose the gene to be delivered to the cells. We have chosen to study p16, p21, p53 and pRb gene transfer using the pCL-retrovirus. Described here are some general concepts and specific results of our work that indicate continued hope for the development of genetically based cancer treatments.

\section{Introduction}

The International Symposium on "Signal Transduction and Gene Expression in Cell Proliferation and Differentiation" held in São Paulo, August 31-September 2, 1998 (following the 17th International Cancer Congress held in Rio de Janeiro, August 24-28, 1998) united clinical and laboratory researchers from all regions of the world. Just as the participants were varied, so were their approaches to understanding and treating cancer. The speakers presented their findings with a common theme of hope for treating and preventing cancer inspired by progress in understanding its origins. Our work presented at these meetings (and described here) also follows this theme: careful, critical understanding of the loss of cell-cycle control in cancer cells and then applying this knowl- edge towards the development of molecular genetic treatment.

The goals of our research are to use gene transfer technologies to deliver cDNA's encoding anti-proliferation proteins to transformed cells. This approach involves basic laboratory research in the areas of cell-cycle control and gene delivery vectors which are then applied to cell culture and animal models of tumor progression. The multifaceted nature of our work can be divided into three interdependent phases: 1) studying and subsequently testing our understanding of cellcycle control in both normal and transformed cells; 2) constructing and continually improving retrovirus-based gene transfer systems, and 3) analyzing both gene transfer and gene function in a relevant animal model system.

Described here are essential concepts of 
cell-cycle control, gene transfer and animal models. Mixed with these fundamental ideas are our own results derived from the study of glioblastoma multiforme cell lines and our hopes for applying our basic, laboratory findings in a clinical setting.

\section{Cell cycle}

The growth and division of normal cells is controlled by a sequence of events known collectively as the cell cycle. The cell cycle can be divided into four distinct stages called G1, S, G2 and M. In the G1 (or first gap) phase, cellular activity is relatively low until a mitogenic signal stimulates a cascade of checks and balances, after which a normal cell will enter the S phase and initiate DNA synthesis. The transition from the $\mathrm{G} 1$ to the $\mathrm{S}$ phase is critical in minimizing the accumulation of genetic damage. In other words, the checks and balances of the G1 phase are used to prevent a cell from entering the $\mathrm{S}$ phase if some genetic damage has occurred. Unregulated entry into the $\mathrm{S}$ phase can allow the replication of genetic alterations, mutations, that are then passed on to daughter cells after the G2 (second gap) phase and $\mathrm{M}$ (mitosis) phase. Although most genetic alterations are inconsequential, corrected by DNA repair mechanisms, or eliminated by the death of the cell due to the loss of a critical gene, rare mutations confer a growth advantage to cells which are further selected for their ability to survive and proliferate under unregulated conditions. Transformed (tumorigenic) cells often, if not always, accumulate alterations in the key genes that regulate the G1 phase. Therefore, a thorough understanding of normal G1 regulation can help to identify the defective cell-cycle control genes in a transformed cell. We then use this information in the development of strategies to arrest the unchecked proliferation.

The control of progression through the G1 phase of the cell cycle depends primarily on the role of the retinoblastoma susceptibil- ity gene product, $\mathrm{pRb}$. If $\mathrm{pRb}$ is maintained in its hypophosphorylated state, progression through G1 is halted. Phosphorylation of $\mathrm{pRb}$ is carried out by a series of cyclindependent kinase (CDK) complexes. The complexes involving CDK4 initiate the phosphorylation of $\mathrm{pRb}$ and the complexes involving CDK2 complete the task and drive the cell cycle past the G1 restriction point (for a complete review, see 1-4). When phosphorylation by CDK complexes is inhibited, the cell no longer arrests in the G1 phase of the cell cycle.

It follows that the inhibition of the G1 CDK complexes can lead to cell-cycle arrest only if $\mathrm{pRb}$ is functional. For example, members of the INK4 class of CDK-inhibitors (CDKIs) (which include p16 ${ }^{\mathrm{INK} 4 \mathrm{~A}}, \mathrm{p} 15^{\mathrm{INK} 4 \mathrm{~B}}$ and $\mathrm{p} 18^{\mathrm{INK} 4 \mathrm{C}}$ proteins) are characterized by their specific binding to CDK4 and CDK6 complexes and subsequent inhibition of $\mathrm{pRb}$ phosphorylation during the G1 phase of the cell cycle (5). Thus, the function of p16 depends on the integrity of both the CDK and $\mathrm{pRb}$ proteins (a combination we call the $\mathrm{p} 16 / \mathrm{pRb}$ pathway). Tumor cells have often lost either p16 or pRb, but less often both, since the loss of just one of these genes is sufficient to permit unchecked transition through early G1. Therefore, the re-introduction of p16 into a tumor cell will arrest growth in G1 only if both the CDK4 (or CDK6) and $\mathrm{pRb}$ proteins are present in their functional forms.

In a related scenario, the p21 (Waf1, Cip1, Sdi1 or CAP20), p27 $7^{\mathrm{Kip} 1}$ and p57 ${ }^{\mathrm{Kip} 2}$ CDKIs have been found to be associated with and to be inhibitory of the activity of all CDK complexes examined, including those involved in the phosphorylation of $\mathrm{pRb}(5,6)$. In normal fibroblasts, p21 serves as an assembly factor in quaternary complexes composed of cyclin-CDK-p21-PCNA. Overexpression of $\mathrm{p} 21$ can arrest cell growth in the G1 phase of the cell cycle by binding to and inhibiting the function of either CDK4 complexes or CDK2 complexes (3). The suc- 
cessful inhibition of tumor cell growth in the G1 phase of the cell cycle by $\mathrm{p} 21$ is dependent on the formation of the complexes with either CDK4 or CDK2 and on the lack of phosphorylation of $\mathrm{pRb}$ by these complexes.

The p21 gene carries a p53-binding sequence in its proximal promoter and can be regulated by p53 (7). Treatment of cells with a variety of DNA-damaging agents leads to increased p53 levels and transactivation of p21 (among other genes), resulting in p53dependent G1 arrest (8). Moreover, in certain situations p21 is expressed independently of p53, e.g., during normal embryo and adult tissue development, following serum stimulation, and during differentiation. Thus, a transcriptional relationship exists between p53 and p21 in which damage to the cell can lead to expression of p21 in a p53-dependent manner (a combination we call the p21/p53 pathway). The p53-mediated arrest of cell growth does not depend exclusively on the expression of p21 (9), such as the induction of genes involved in apoptosis (10-12). The reintroduction of p53 into tumor cells may arrest growth by mechanisms not involving p21.

One function of $\mathrm{pRb}$ is to bind to and sequester proteins which are required for cell-cycle progression, among them E2F. These proteins are released from the bound state upon the phosphorylation of $\mathrm{pRb}$. It follows that $\mathrm{pRb}$ functions downstream of the CDK complexes. Moreover, pRb has some CDK-independent functions, such as the repression of some promoters and RNA pol III activity (13-18). Therefore, the reintroduction and expression of $\mathrm{pRb}$ into tumor cells may arrest growth by mechanisms that are independent of CDK complex formation.

Our studies have focused on the outcome of replacing a defective $\mathrm{p} 16, \mathrm{p} 21, \mathrm{p} 53$ or $\mathrm{pRb}$ gene with the wild-type counterpart in glioblastoma cell lines. By the above descriptions, p16 should act as an inhibitor of proliferation specific for cells which express $\mathrm{pRb}$.
On the other hand, p21, p53 and pRb each perform a variety of functions that are not necessarily dependent on disruption of CDK activity and should act as general inhibitors of proliferation. Therefore, understanding the status of endogenous cell-cycle control genes should be a useful tool in predicting the efficacy of replacing one of the defective genes.

While working in the laboratory of Dr. Martin Haas at the University of California, San Diego, we assayed for the integrity of the $\mathrm{p} 16 / \mathrm{pRb}$ and $\mathrm{p} 21 / \mathrm{p} 53$ pathways in a series of human glioblastoma multiforme cell lines (19). We used standard techniques to analyze genomic DNA, mRNA and protein status of the p16, p21, p53, and pRb genes. Among the eight human glioblastoma lines analyzed, all were defective in the $\mathrm{pRb} /$ p16 cell-cycle control pathway due to alterations in pRb, p16 or both, while 7 of 8 $(87.5 \%)$ of the lines had a defective $\mathrm{p} 53 / \mathrm{p} 21$ cell-cycle control pathway due to alteration at the p53 locus. Our results show that among the eight glioblastoma cell lines studied, seven were defective in both the $\mathrm{pRb} /$ p16 and the p53/p21 pathways. The next step in our studies was to use a retrovirusbased gene delivery system to replace these defective genes and assess the outcome. In order to accomplish this, we have carefully examined the available technologies of gene replacement and expression.

\section{Technologies of gene replacement}

The transfer of a therapeutic gene to a target cell has, for many years now, been proposed as a treatment for a variety of genetic disorders. Such treatments could permit the production of a faulty or missing protein in chronic, inherited diseases such as cystic fibrosis or hemophilia. In an acquired disease like cancer, a gene may be introduced into the tumor cell which would direct its demise by an immunologic attack, interruption of DNA synthesis, blockage of an- 
giogenesis, sensitization to radio- or chemotherapy, or induction of cell-cycle arrest genes. Alternatively, a gene like that for multidrug resistance may be introduced into the bone marrow in order to protect it against very high doses of traditional chemotherapeutic agents (20). Although these concepts continue to appear promising, the critical component for the success of these techniques will be the development of vectors to deliver the therapeutic gene and drive its expression in the target cells (for excellent reviews, see 20-22).

What are the factors that make a gene delivery system ideal for replacing a defective gene? The answer is dependent upon the function of the gene and the cell type in which it is expected to perform. For example, in the treatment of cancer, a gene which drives the cell towards apoptosis needs to be expressed for only a short time. In this situation, integration of exogenous DNA into the host genome is not necessary since cells will not have a chance to divide, and therefore there is no need for expression in daughter cells. In addition, the vector must be specific for the tumor cells in order to avoid destruction of healthy cells. However, in the treatment of a chronic disease, such as hemophilia, the delivered coagulation factor must be expressed for a long period of time in order to give the patient continued protection from uncontrolled bleeding. Here integration of the exogenous genetic material is advantageous since it would help provide the therapeutic gene to descendant cells. Gene transfer for production of a secreted factor may be achieved in a variety of tissue types, reducing problems associated with targeting a specific tissue.

Although an immunologic response to the therapeutic gene may be desired (as is the case for IL-2 delivery to cancer cells (20)), an immune response to the delivery vehicle itself is undesirable. An immunologic attack by complement may prevent the vector from reaching its target cells. In addition, humoral immunity gained by exposure to the vector may preclude multiple rounds of treatment. Therefore, immunologic transparency is a desirable feature in a gene transfer system. Other considerations include the titer of the vector, the rate of division and the location of the target cells, specific transfer to only the target cells, as well as ex vivo versus in situ gene transfer (20-22). In short, no single existing system can be used to satisfy all possible requirements and, therefore, vectors must be designed with their application in mind.

Gene delivery systems fall into two broad categories: virus- and non-virus-based. The virus-based systems include viruses with a DNA genome, such as adenovirus, adenoassociated virus and herpes virus (among others) as well as viruses with an RNA genome, such as retroviruses in the murine leukemia virus family or lentivirus in the HIV family. Non-virus-based systems include plasmids and cosmids which are delivered by a variety of transfection techniques. Discussed here are some of the features, advantages and disadvantages of each of these systems.

Non-viral gene delivery vectors (naked DNA vectors) are delivered by means of calcium phosphate precipitation, lipofection or electroporation. In general, these methods are not specific for the target cell, do not elicit an immune response, and are not dependent upon target cell proliferation or location (when re-introduced into the patient). The vectors themselves are generally based on bacterial plasmids or cosmids. Thus, the vector can carry any size gene, any promoter, and sequences to direct episomal replication not only in the target cell, but also in bacteria. This flexibility allows for relatively simple manipulation by standard recombinant DNA techniques as well as relatively easy large-scale production of the therapeutic agent. This technology is generally limited to ex vivo gene transfer, which may be advantageous. If stable transfectants are re- 
quired, the cells may be selected in tissue culture for vector-encoded resistance to an antibiotic, commonly genetycin (G418). However, these vectors do not generally integrate into the host genome, making longterm expression difficult in the progeny of the original target cells in the absence of selection. If integration is desired, the DNA vector may encode sequences for homologous recombination (20-22).

Many viruses, with either a DNA or RNA genome, are being developed as gene delivery vectors. The prototypical example of a DNA virus as a vector of gene transfer is the adenovirus. Some advantages are that the adenovirus genome is relatively large and therefore tolerates a larger insert, high titer virus production is routinely achieved, and the viral DNA does not integrate in the host genome. The lack of integration avoids any problems associated with insertional mutagenesis, the interruption of a vital host gene or the accidental activation of an undesirable gene (e.g. an oncogene). However, the lack of integration also means that the viral expression is limited to the cells originally infected since progeny will lose the viral sequences. A serious problem with adenovirus vectors is that the viral proteins induce a strong host immune response, making subsequent infections difficult (20-22).

Adenoviral vectors are well suited to the treatment of cancer if the vector is used to introduce a suicide gene. In this scenario, a pro-apoptotic gene is transferred with the intent of sensitizing (or outright killing) the target cells. With the transfer of p53, the cells would then be more sensitive to ionizing radiation and chemotherapeutic agents which damage DNA (11). However, the host immune response may limit repeated treatment with the same virus. In our opinion, repeated treatment would be necessary with any viral system in order to reach a maximum number of target cells. We have elected not to use adenovirus as a gene transfer vector in an attempt to avoid potential com- plications associated with host immune response.

We have chosen to work with retroviruses since this system, although not perfect, does have many features which can be developed for effective gene transfer (23). The RNA genome of the retrovirus undergoes reverse transcription to produce a doublestranded proviral genome that is then integrated randomly into the host genome. However, the vectors used by us and other laboratories produce progeny virus only under very specific conditions.

For safety reasons, it is desirable that the viral vector be incapable of replicating itself in the target cells. This prevents a widespread infection and helps to limit the infection and viral expression to the target cells. In the laboratory, the viral genome is split into two separate vectors. The packaging vector encodes the structural and functional viral genes but lacks a packaging signal, thus these transcripts are not encapsidated. The second vector encodes the therapeutic gene, but no genes that can support viral proliferation. The virus that is produced when the two vectors are co-expressed in a packaging cell is made up of the normal complement of viral proteins and an RNA genome capable of therapeutic gene transfer, integration and expression, but not of viral proliferation. Such viral progeny are called replication defective (23).

The use of a retrovirus for therapeutic pursuits has three potential pitfalls. The first is the possibility of insertional mutagenesis, the second is the danger of producing replication-competent virus due to recombination with a naturally occurring endogenous retrovirus, and the third the potential of activating an endogenous retrovirus. Although these are each serious considerations when using a retrovirus for gene transfer, we feel that our proposed application of the virus will successfully avoid these complications.

It is our opinion that retrovirus systems, on the whole, are the best choice for gene 
delivery. They are better known, with welldefined systems for production and safety and they elicit a minimal immune response. Our primary goal for developing retroviral vectors is the continued study of cell-cycle control genes in a basic laboratory setting. In this environment, these vectors are quite safe when handled properly since they are easily destroyed by $10 \%$ bleach, soap, and the complement system (23). Thus, laboratory spills and accidental exposure are easily contained.

Our long-term goal of using these vectors in a clinical application is specifically designed to minimize the dangers of retrovirus integration or recombination. We propose that the vectors will be used to transfer the wild-type counterpart of a defective cellcycle control gene in order to eliminate the proliferating cell by apoptosis or senescence. Thus the infected cells would be eliminated, along with the therapeutic virus.

A critical factor for the successful completion of the proposed application is the use of the pCL retrovirus system (24). The pCL system permits the production of high titer viruses which encode cytostatic genes, like p53. The use of the late-passage 293 cell line permits efficient packaging of the retrovirus since this line is easily transfected by the calcium-phosphate precipitation method. As measured by staining for exogenous B-galactosidase activity, we can routinely achieve transfection efficiencies in the range of 40$60 \%$ of cells. In addition, the fusion of the CMV immediate early promoter with the $5^{\prime}$ LTR of the virus strongly promotes expression of the viral mRNA, thus enhancing titer. The packaging system is also fast since only a transient transfection of the packaging information along with the $\mathrm{pCL}$ vector is required. This minimizes the effects of the virus-encoded gene on the 293 cells, again increasing the titer of viruses carrying a cytostatic gene (24). We routinely achieve virus titers of $10^{6}$ colony-forming units per $\mathrm{ml}$ $(\mathrm{cfu} / \mathrm{ml})$ of supernatant in a production sys- tem that requires just overnight incubation. Thus the pCL retrovirus system is a rapid, reliable, and efficient method to transfer tumor suppressor genes in tissue culture.

\section{0 ur results using the $\mathrm{pCL}$ retrovirus to transfer cell-cycle control genes to glioblastoma cell lines}

We have reported the use of the $\mathrm{pCL}$ retrovirus system to replace the $\mathrm{p} 16, \mathrm{p} 21$, $\mathrm{p} 53$, and $\mathrm{pRb}$ cDNAs in a series of human glioblastoma cell lines (19). After confirming the expression of the transduced gene, we assessed the alteration in cellular proliferation by colony formation assays, trypanblue exclusion, and changes in cellular morphology. The details of these results are a bit cumbersome and may fall outside the scope of this discussion. Instead I will address the general findings that illustrate the successes and shortcomings of pCL-mediated gene transfer.

To assess the effect of anti-proliferation genes, typically the colony formation assay is used. In this assay, the glioblastoma cells were infected with one of the $\mathrm{pCL}$ viruses encoding $\mathrm{p} 16, \mathrm{p} 21, \mathrm{p} 53$ or $\mathrm{pRb}$ and then selected for G418 resistance conferred by the virally encoded neomycin phosphotransferase gene. If the cell-cycle control gene was effective in arresting proliferation, few or no colonies formed. However, cells infected by the parental pCL virus (not encoding an anti-proliferation gene) would produce many G418-resistant colonies. Recall that we predict exogenous p16 to be effective in reducing the number of colonies formed only when the cell line expresses endogenous $\mathrm{pRb}$. In contrast, we expect $\mathrm{p} 21$, p53 and pRb to act as general inhibitors of colony formation due to their multiple molecular targets.

The expression of pCLp16 virus reduced the colony formation of the $U 87^{\mathrm{p} 16-/ \mathrm{Rb}+}$, $\mathrm{LW}^{\mathrm{p} 16-/ \mathrm{Rb} \mathbf{b}^{+}}$and $\mathrm{U} 25^{\mathrm{p} 16-/ \mathrm{Rb}+}$ cell lines by essentially $100 \%$ (19). In contrast, the lines 
D384 ${ }^{\mathrm{p} 16+/ \mathrm{pRb}-}$ and SF126 $6^{\mathrm{p} 16-\mathrm{pRb}-}$ were unaffected by the expression of exogenous p16. Hence, the $\mathrm{pRb}^{+}$cells were sensitive to $\mathrm{p} 16$ mediated suppression as predicted by the model of G1 cell cycle control.

Replacement of the p21 gene by infection with pCLp21 virus resulted in significant growth suppression of the SF126 $6^{\mathrm{p} 21-/ \mathrm{p} 53-}$, U87 ${ }^{\mathrm{p} 21+/ \mathrm{p} 53 \mathrm{wt}}$ and U251 ${ }^{\mathrm{p} 21-\mathrm{p} 53 \mathrm{mt}}$ glioblastoma cells. Moderate suppression was observed in LW5 ${ }^{\text {p21-p53- }}$ cells, while D384 ${ }^{\text {21-/p53mt }}$ cells were not affected by the expression of the transferred p21 gene (19). p21, which is considered to be a universal inhibitor of CDKs $(7,25)$, was not uniformly able to suppress growth of the glioblastoma lines.

Transfer of wild-type p53 or pRb to the glioblastoma lines could suppress colony formation in all lines tested. However, the efficacy of growth suppression was varied and did not appear to correlate with the endogenous status of the four genes analyzed.

Similar results were obtained in a different assay for cell proliferation, trypan-blue exclusion. This assay involves counting the number of living cells (which remain unstained) each day for a week following infection, but without G418 selection. We observed inhibition of proliferation by p 16 only in cells with endogenous $\mathrm{pRb}$. We also observed a pattern of inhibition by $\mathrm{p} 21, \mathrm{p} 53$ and $\mathrm{pRb}$ consistent with the colony formation assays.

In general, predicting the outcome of gene replacement was possible, but difficult. While p16 transfer yielded the clearest and most consistent results, p21, p53 and pRb did act in a general manner, even if a bit inconsistent.

The morphology was altered in glioblastoma cells following infection with retroviruses encoding tumor suppressor genes. Nearly all the cells that survived pCLp16 and pCLp21 virus infection showed an aberrant morphology and were clearly distinguishable from their parental cells by their flat appearance, large size (size increases of $\sim 5$-10-fold), multiple nucleoli and easily identifiable cytoplasmic vacuoles. Induction of flat cells or of a bipolar morphology was never observed following the infection of glioblastoma cells with control virus.

Glioblastoma cell infection with pRbencoding virus did not result in the appearance of flat cells, but the size of the surviving colonies and the number of cells in each colony were reduced. Expression of exogenous p53 produced a small increase in the number of cells with a flat phenotype. When p53-induced flat cells were observed, they were localized preferentially around the edges of the colonies, in contrast to a homogeneous distribution of flat cells in colonies grown out from p16- or p21-virus-infected cells, consistent with previous observations (26-28).

To summarize, the alterations in morphology occurred in a manner consistent with the results of the colony formation and trypan-blue exclusion assays. Overall, the combined results of these experiments show that predicting the outcome of gene transfer using the status of endogenous gene expression as an indicator is possible, but difficult. The efficacy of halting cell proliferation was better with p16 and p21 (in the cases where these genes were effective) as compared to the general, yet milder response of cell lines to the transfer of $\mathrm{p} 53$ and $\mathrm{pRb}$.

\section{Animal models}

Animal models for tumor formation can be extremely informative in terms of not only gene function, but also gene transfer. Observing gene function in vivo is the ultimate test of its action since in vitro and tissue culture systems may produce system-specific artifacts. The most common animal model of tumor formation involves the injection of transformed cells under the skin of nude mice, the xenograft tumor model. The nude mice lack a functional immune system, thus 
injected cells from most any source are not eliminated by a host $v s$ graft response. The cells may be from most any type of tumor, thus a human lung tumor cell line may grow in the xenograft model. This means that the tumor is not in its host species, not in its normal location of growth, and in an environment lacking an immune response. Although this system can yield many interesting results, we feel it is much too distant from our proposed use of retroviral gene transfer to tumor cells.

The model that we have successfully used involves the transfer of a cell cycle control gene to rat-derived glioblastoma cell lines, injecting the infected cells into the brain of a normal rat, and comparing the growth of the treated cells to that of untreated control cells. Tumorigenic cells are infected in tissue culture before transfer to the animal, an ex vivo infection protocol. The cells are placed in the rat brain using stereotactic injection, a system which precisely locates the needle at a desired coordinate within the brain. Following surgery, the rats are kept for 45 to 60 days to allow tumor formation. The tumors are recovered and analyzed in histologic sections.

In collaboration with Dr. Gerald Silverberg's laboratory at Stanford University, the p21 cDNA was transferred to RT-2 rat glioma cells using the $\mathrm{pCL}$ retroviral delivery system, and tumor growth was dramatically reduced as compared to the control tumor infected with the parental virus (29). This shows that the function of $\mathrm{p} 21$ was effective for the arrest of tumor proliferation in vivo. However, the ex vivo infection protocol may have allowed the gene to function before injection into the rats. Therefore, we are striving to develop an in situ infection protocol.

We are confident that we have established a foundation on which to build our in situ infection protocol here at the University of São Paulo. Working in collaboration with Dr. José Cipolla Neto, we have infected C6 rat glioma cells with either the p16 or the parental pCL virus. Our unpublished data show that the p16 virus was extremely efficient in arresting tumor formation as compared to the control virus. Although an ex vivo infection protocol was used, we are now in a position to begin experimenting with in situ delivery of virus to a pre-established tumor in the rat brain.

\section{Clinical trials}

In the United States, 232 clinical trials for gene therapy had been approved as of February, 1998 and over 300 worldwide. In the US, $69 \%$ of the trials are for the treatment of cancer, $8 \%$ for cystic fibrosis, and $11 \%$ for AIDS $(21,30)$. So far there have been no true successes for the use of gene therapy for the treatment of these diseases. The problem, as we see it, has two principal components. First is the design of the vector used and second is the limitations placed on most clinical trials. Vector design is continually improving and we are confident that vectors will soon be available which have reliable, high level expression of the therapeutic gene. In addition, the choice of the therapeutic gene must be (and is being) addressed as part of the vector design.

The design of the clinical trials themselves typically faces a fundamental problem in that experimentation is not permitted in cases that are less than grave. This puts the trial at a disadvantage since the therapy is applied only at an extremely late stage of disease progression. In the case of cancer, late stages may be inoperable and are often associated with metastatic lesions. In practice, genetic therapy of cancer would be applied at earlier stages, therefore clinical trials should reflect this proposed use if successful results are to be obtained.

\section{Perspectives}

As stated above, our primary goal for the 
use of pCL-mediated gene transfer is for the evaluation of the function of cell-cycle control genes. To this end we have established a firm foundation on which to build studies of $\mathrm{p} 16, \mathrm{p} 21, \mathrm{p} 53$ and $\mathrm{pRb}$ function.

We strongly feel that attempting to predict transgene function in a transduced cell is of great importance. However, the variety of alterations in endogenous gene status may be better assessed by technologies that allow for the screening of many genes in a very rapid manner. Such assays may be, and are, carried out using commercially available membranes or even silicon chips to which specific nucleotide segments for a variety of genes have been permanently linked (31). Labeled RNA fragments are hybridized with these in order to assess the expression and/or mutation of genes. These systems can analyze the expression and/or mutation of genes much more efficiently than the standard techniques which we have employed. Knowing the status of hundreds of genes would certainly contribute to better prediction of a cell's capacity to permit the function of a therapeutic transgene. Moreover, understanding the interaction of these genes is critical for identifying therapeutic cDNAs. We are confident that up-and-coming technologies will make assessing alterations in $\mathrm{G} 1$ control genes fast and comprehensive.

We are actively trying to improve the pCL retrovirus system so that better assays of gene function and transfer would be possible. We suggest that better assays for the action of anti-proliferation genes would yield more telling information about gene function. This may involve increasing the levels and duration of gene expression, the method by which proliferation is monitored, and the method by which infected cells are identified. In this way we will continue our studies of the function of p16, p21, p53 and $\mathrm{pRb}$. We also hope that such improvements of the viral system would also make a more critical analysis of gene transfer feasible.
In general, we envision the use of gene transfer as a combination, not a replacement, of existing cancer therapies. Immediately following removal of a glioblastoma tumor, for example, the surgical field would be flooded with virus with the intent of infecting residual tumor cells and inducing their demise. The application of chemo- or radiation therapy could act in concert with the therapeutic gene, especially true for p53 which induces apoptosis in response to DNA damage (32). Of course, this protocol may be applicable to a wide range of tumor types so long as the virus and its encoded gene are well suited for the targeted cell type. No gene delivery system can reach $100 \%$ of the target cells and we do not expect gene therapy to be effective in reducing the mass of an existing solid tumor. However, the introduction of a therapeutic gene in combination with surgery, chemotherapy, and radiation therapy is a promising, yet unrealized, possibility.

Is Brazil ready for clinical trials of gene therapy?

Is the pCL system ready?

These are tough questions to which we do not have a firm answer. However, the answer will involve the joint efforts of basic scientists and clinicians. The study of cancer involves many disciplines (such as cell biology, molecular biology and virology) for which basic scientists are well suited. However, the development of treatment protocols requires close cooperation between laboratory scientists and clinicians. The clinicians are uniquely qualified for establishing clinical trial designs, from evaluation of safety and ethical issues to patient suitability in clinical trials and, of course, administering the treatment to the patient. We hope that experience and expertise can be shared not just at occasional symposia, but on a daily, collaborative basis. Therefore, answering these questions will depend upon the collaboration of basic scientists and clinicians. 


\section{Acknowledgments}

We wish to thank Drs. Mari Armelin and Claudio Joazeiro for their invitation to attend the International Symposium on "Signal Transduction and Gene Expression in
Cell Proliferation and Differentiation" and for the opportunity to write this review. We also wish to thank Drs. Robert Naviaux and Martin Haas of the University of California, San Diego, for their support and contributions to our past and present efforts.

\section{References}

1. Hunter T (1993). Braking the cycle. Cell, 75: 839-841.

2. Hunter T \& Pines J (1994). Cyclins and cancer. II: Cyclin D and CDK inhibitors come of age. Cell, 79: 573-582.

3. Sherr CJ (1994). G1 phase progression: cycling on cue. Cell, 79: 551-555.

4. Sherr CJ (1995). Mammalian G1 cyclins and cell cycle progression. Proceedings of the Association of American Physicians, 107: 181-186.

5. Grana X \& Reddy EP (1995). Cell cycle control in mammalian cells: role of cyclins, cyclin dependent kinases (CDKs), growth suppressor genes and cyclin-dependent kinase inhibitors (CKIs). Oncogene, 11: 211-219.

6. el-Deiry WS, Tokino T, Waldman T, Oliner JD, Velculescu VE, Burrell M, Hill DE, Healy E, Rees J L, Hamilton SR, Kinzler KW \& Vogelstein B (1995). Topological control of p21WAF1/CIP1 expression in normal and neoplastic tissues. Cancer Research, 55: 2910-2919.

7. el-Deiry WS, Tokino T, Velculescu VE, Levy DB, Parsons R, Trent J M, Lin D, Mercer WE, Kinzler KW \& Vogelstein B (1993). WAF1, a potential mediator of p53 tumor suppression. Cell, 75: 817-825.

8. Dulic V, Kaufmann WK, Wilson SJ, Tlsty TD, Lees E, Harper JW, Elledge SJ \& Reed SI (1994). p53-dependent inhibition of cyclin-dependent kinase activities in human fibroblasts during radiation-induced G1 arrest. Cell, 76: 1013-1023.

9. Macleod KF, Sherry N, Hannon G, Beach D, Tokino T, Kinzler K, Vogelstein B \& J acks T (1995). p53-dependent and independent expression of p21 during cell growth, differentiation, and DNA damage. Genes and Development, 9: 935-944.

10. Miyashita T, Harigai M, Hanada M \& Reed J C (1994). Identification of a p53-dependent negative response element in the bcl2 gene. Cancer Research, 54: 3131-3135.

11. Miyashita T, Krajewski S, Krajewska M, Wang HG, Lin HK, Liebermann DA, Hoffman B \& Reed J C (1994). Tumor suppressor p53 is a regulator of bcl-2 and bax gene expression in vitro and in vivo. On- cogene, 9: 1799-1805.

12. Miyashita T \& Reed J C (1995). Tumor suppressor p53 is a direct transcriptional activator of the human bax gene. Cell, 80 : 293-299.

13. Chow KN, Starostik P \& Dean DC (1996). The Rb family contains a conserved cyclindependent-kinase-regulated transcriptional repressor motif. Molecular and Cellular Biology, 16: 7173-7181.

14. White RJ, Trouche D, Martin K, J ackson SP \& Kouzarides T (1996). Repression of RNA polymerase III transcription by the retinoblastoma protein. Nature, 382: 8890.

15. Chow KN \& Dean DC (1996). Domains A and $B$ in the $R b$ pocket interact to form a transcriptional repressor motif. Molecular and Cellular Biology, 16: 4862-4868.

16. Sellers WR, Rodgers JW \& Kaelin WG (1995). A potent transrepression domain in the retinoblastoma protein induces a cell cycle arrest when bound to E2F sites. Proceedings of the National Academy of Sciences, USA, 92: 11544-11548.

17. Weintraub SJ , Chow KN, Luo RX, Zhang SH, He S \& Dean DC (1995). Mechanism of active transcriptional repression by the retinoblastoma protein. Nature, 375: 812815.

18. Adnane J , Shao Z \& Robbins PD (1995). The retinoblastoma susceptibility gene product represses transcription when directly bound to the promoter. J ournal of Biological Chemistry, 270: 8837-8843.

19. Costanzi-Strauss $E$, Strauss $B E$, Naviaux RK \& Haas M (1997). Restoration of cellgrowth arrest by p16, p21, pRb and p53 is dependent on the integrity of the endogenous cell-cycle control pathways in human glioblastoma cell lines. Experimental Cell Research, 238: 51-62.

20. Culver KW, Van Gilder J, Carlstrom T, Prados M \& Link J r CJ (1995). Gene therapy for brain tumors. In: Chang PL (Editor), Somatic Gene Therapy. CRC Press, Inc., Boca Raton, FL.

21. Anderson WF (1998). Human gene therapy. Nature, 392 (Suppl): 25-30.

22. Verma IM \& Somia N (1997). Gene therapy - promises, problems and prospects. Nature, 389: 239-242.

23. Coffin JM, Hughes $\mathrm{SH} \&$ Varmus HE (1997). Retroviruses. Cold Spring Harbor Laboratory Press, Plainview, New York.

24. Naviaux RK, Costanzi E, Haas M \& Verma I (1996). The pCL vector system: rapid production of helper-free, high-titer, recombinant retroviruses. J ournal of Virology, 70: 5701-5705.

25. Xiong $Y$, Hannon $G$ J , Zhang $H$, Casso $D$, Kobayashi R \& Beach D (1993). p21 is a universal inhibitor of cyclin kinases. Nature, 366: 701-704.

26. Van Meir EG, Kikuchi T, Tada M, Li H, Diserens AC, Wojcik BE, Huang HJ, Friedmann $\mathrm{T}$, de Tribolet $\mathrm{N} \&$ Cavenee WK (1994). Analysis of the p53 gene and its expression in human glioblastoma cells. Cancer Research, 54: 649-652.

27. Van Meir EG, Roemer K, Diserens AC, Kikuchi T, Rempel SA, Haas M, Huang HJ Friedmann $\mathrm{T}$, de Tribolet $\mathrm{N} \&$ Cavenee WK (1995). Single cell monitoring of growth arrest and morphological changes induced by transfer of wild-type p53 alleles to glioblastoma cells. Proceedings of the National Academy of Sciences, USA, 92: 1008-1012.

28. Horton LE, Qian Y \& Templeton DJ (1995). G1 cyclins control the retinoblastoma gene product growth regulation activity via upstream mechanisms. Cell Growth and Differentiation, 6: 395-407.

29. Hsiao M, Tse V, Carmel J, Costanzi E, Strauss B, Haas M \& Silverberg GD (1997). Functional expression of p21(WAF1/Cip1) gene in rat glioblastoma cells suppresses tumor growth in vivo and induces radio sensitivity. Biochemical and Biophysical Research Communications, 233: 329-335.

30. ORDA Report: Human Gene Therapy Protocols (1998). Office of Recombinant DNA Activities. NIH, Bethesda, MD.

31. J ohnston M (1998). Gene chips: array of hope for understanding gene regulation. Current Biology, 8: 171-174.

32. Ko LJ \& Prives C (1996). p53: puzzle and paradigm. Genes and Development, 10: 1054-1072. 